Post-print of: Vélez, P., et al. "Microwave microfluidic sensor based on a microstrip splitter/combiner configuration and split ring resonators (SRRs) for dielectric characterization of liquids" in IEEE sensors journal (Ed. Institute of Electrical and Electronics Engineers), vol. 17, issue 20 (2017), p. 6589-6598.

DOI 10.1109/JSEN.2017.2747764.

Cop. 2017 IEEE. Personal use of this material is permitted. Permissions from IEEE must be obtained for all other uses, in any current or future media, including reprinting/republishing this material for advertising or promotional purposes, creating new collective Works, for resale or redistribution to servers or lists, or reuse of any copyrighted component of this work in other Works.

The final version is available at http://ddd.uab.cat/record/188171 under a cop. All rights reserved license 


\title{
Microwave Microfluidic Sensor based on a Microstrip Splitter/Combiner Configuration and Split Ring Resonators (SRR) for Dielectric Characterization of Liquids
}

\author{
Paris Vélez, Member, IEEE, Lijuan Su, Member, IEEE, Katia Grenier, Member, IEEE, Javier \\ Mata-Contreras, David Dubuc, Member, IEEE, and Ferran Martín, Fellow, IEEE
}

\begin{abstract}
A microwave microfluidic sensor for dielectric characterization of liquids in real time is presented in this paper. The sensor is implemented in microstrip technology and consists of a symmetric splitter/combiner configuration loaded with a pair of identical split ring resonators (SRRs) and microfluidic channels placed on top of them (gap region). The sensor works in differential mode and sensing is based on frequency splitting. Thus, if the structure is unloaded or if it is symmetrically loaded with regard to the axial plane, only one transmission zero (notch) in the frequency response appears. However, if the axial symmetry is disrupted (e.g., by the presence of different liquids in the channels), two transmission zeros arise, and the difference in magnitude (notch depth) and frequency between such transmission zeros is indicative of the difference in the dielectric properties (complex dielectric constant). A circuit schematic, including transmission line sections to describe the distributed components, lumped elements to account for the SRRs and their coupling to the lines and lumped elements to model the liquid properties, is presented and validated. After proper calibration, the functionality of the proposed sensor is demonstrated by measuring the complex permittivity in solutions of deionized (DI) water and ethanol as a function of the ethanol content.
\end{abstract}

Index Terms - Microwave sensors, differential sensors, microfluidics, dielectric characterization of liquids, permittivity measurements, microstrip technology, split ring resonator (SRR).

\section{INTRODUCTION}

E lectrically small resonators coupled to a host transmission lines have been used in a wide variety of radiofrequency (RF) and microwave applications (see [1],[2] and references therein), including sensors. In microwave sensors based on resonant elements (either coupled to a host line or not), the usual sensing principle relies on the variation of the

This work was supported by MINECO-Spain (projects TEC2013-40600-R and TEC2016-75650-R), Generalitat de Catalunya (projects 2014SGR-157 and TECSPR15-1-0050), Institució Catalana de Recerca i Estudis Avançats (who awarded Ferran Martín), and by FEDER funds. The work of L. Su was supported by the China Scholarship Council under Grant 201306950011.

P. Vélez, L. Su, J. Mata-Contreras and F. Martín are with GEMMA/CIMITEC, Departament d'Enginyeria Electrònica, Universitat Autònoma de Barcelona, 08193 Bellaterra, Spain (e-mail: Ferran.Martin@uab.es).

K. Grenier and D. Dubuc are with MH2F, LAAS-CNRS, 7th, Avenue du Colonel Roche BP 5420031031 Toulouse, Cedex 4, France (e-mail: Grenier@laas.fr). resonance frequency, phase or quality factor produced by the variable to be sensed (measurand) [3]-[24]. Such sensors are subjected to cross sensitivities, particularly those derived from environmental changing conditions (temperature, moisture, etc.). Hence, ambient factors are potential causes of drifts in the output variables not related to variations in the measurand [19],[25].

To improve the robustness of microwave sensors against environmental factors, differential-mode operation is a possible solution [20]-[22],[26],[27]. In differential sensors, two sensing elements are used and the input variable is the difference in the value of the measurand at each sensing element. Differential sensors can be used as comparators as well, where a sample under test (SUT) is compared to a reference sample in order to detect possible defects or abnormalities (manifested by a finite value of the differential output variable). Nevertheless, for the characterization of samples based on differential measurements the reference sample must have well known properties, in order to infer the magnitude (value) of the measurand in the SUT.

A similar approach to differential sensing was proposed in [28], where symmetry properties in transmission lines loaded with a single (symmetric) resonant element were considered for sensing purposes (see an exhaustive review of the approach in [2],[29]). In these sensors, the line is loaded with a symmetric resonator with its symmetry plane perfectly aligned with the symmetry plane of the line. By choosing a resonator exhibiting an electric wall at its symmetry plane at the fundamental resonance (the one of interest), the resonator is not coupled to the line (provided the symmetry plane of the lines is a magnetic wall, as occurs in the usual transmission lines, e.g., microstrip, coplanar waveguide, etc.). The lack of coupling is due to the perfect cancellation of fields (electric and magnetic) generated by the line in the resonator area, consequence of the different electromagnetic nature of the symmetry planes of line and resonator (one a magnetic wall and the other one an electric wall) [2],[29]. However, by truncating symmetry, this perfect cancellation no longer holds, the resonator is excited, and the line exhibits a transmission zero (notch) at the fundamental resonance. The magnitude of the notch gives a measure of the level of asymmetry and hence it can be used for the measurement of variables related to the 
asymmetry (typically spatial variables such as displacement or velocity [30]-[37]). The sensing principle is the modulation of the coupling level between the resonator and the line caused by symmetry disruption (another type of sensors that also exploits coupling modulation and use multiple resonant elements to modulate a harmonic signal have been applied to the measurement of instantaneous angular velocities [38],[39]). Interestingly, the symmetry is invariant under changing environmental conditions, and, therefore, this type of sensors is robust against cross sensitivities caused by external factors. Sensors with good sensitivity, linearity and dynamic range (by considering the notch depth in $\mathrm{dB}$ as the output variable) have been demonstrated, but the notch depth at small perturbations may be obscured by noise effects (especially critical if the sensing elements are wirelessly connected to the sensor electronics).

A different sensing strategy, also related to symmetry, is frequency splitting [29],[40]-[45]. In this case, a transmission line is loaded with a pair of resonant elements in a symmetric configuration. If symmetry is preserved, a single transmission zero at the resonance frequency of the loading elements arises. However, if symmetry is truncated, e.g., by loading the resonant elements by means of asymmetric dielectric loads, two notches appear as consequence of the different resonance frequencies of the loaded resonators. The difference in frequency and magnitude (notch depth) between the notches is directly related to the level of asymmetry and can be used for sensing purposes.

A limiting aspect of these frequency splitting sensors is sensitivity degradation for small perturbations (asymmetries). This is due to inter-resonator coupling, inevitable if the resonators are close enough. To solve this problem, a cascaded configuration was presented in [46]. Namely, by placing the resonant elements at different positions along the line (e.g., separated by $\lambda / 2, \lambda$ being the guided wavelength at the resonance frequency), inter-resonator coupling is prevented.

An alternative solution is the splitter/combiner configuration [47],[48], where a pair of sufficiently separated parallel transmission lines (each one loaded with a resonant element) prevents from inter-resonator coupling as well. If the structure is symmetric a single transmission zero arises, contrary to the pair of notches that result by asymmetrically loading the resonators. However, for asymmetrically loaded splitter/combiner structures, the pair of transmission zeros are due, in general, to an interfering phenomenon [47]. Such zeros are not given by the resonance frequencies of the resonant elements since at such frequencies transmission is prevented in one of the lines but not in the other line. Under these conditions, sensor sensitivity is degraded at small perturbations as well. However, this limitation can be solved by appropriately choosing the length of the transmission line sections of the splitter/combiner. Specifically, the sensitivity is optimized by translating at the $\mathrm{T}$-junctions a ground connection resulting from the impedance of the resonant elements at the corresponding resonance frequency (see [47]). By this means, by loading one of the resonators by means of a dielectric load, thus causing an asymmetry in the structure, a notch always appears at the resonance frequency of the unloaded resonator (regardless of the characteristics of the dielectric load present on the other one). The reason is that such transmission zero frequency is not consequence of interference, but simply given by the resonance frequency of the unloaded resonator, where a short-circuit is seen at the plane of the input and output $\mathrm{T}$-junctions. If the sensor works by using a reference sample, or load, in one of the resonators, the resonance frequency of the loaded resonator is the one that must be used for the calculation of the optimum length of the splitter/combiner configuration.

In this paper, a differential microwave microfluidic sensor based on the previous splitter/combiner concept, useful for the characterization of the complex permittivity of liquid samples, is proposed. The sensor is implemented in microstrip technology and the loading elements are split ring resonators (SRRs). The paper is organized as follows. The structure of the proposed microfluidic sensor, including the microwave circuitry (splitter/combiner configuration plus resonant elements) and the mechanical and fluidic parts, is reported in Section II. In this section, the circuit model of the proposed sensor is presented and validated, as well. The definition of the materials, sensor fabrication and calibration are summarized in section III. In this section it is also shown that the dielectric constant and losses of the liquids can be taken into account in the model by including an additional capacitance and resistor. In section IV, the fabricated prototype microfluidic sensor is validated by applying it to the measurement of the complex permittivity of DI water/ethanol solutions. A discussion and a comparison between the presented differential-mode approach and recent microwave microfluidic sensors for dielectric characterization of DI water/ethanol solutions are included in section V. Finally, the main conclusions of the work are highlighted in section VI.

\section{STRUCTURE OF THE PROPOSED SENSOR, CIRCUIT MODEL AND VALIDATION}

The proposed microwave microfluidic sensor is composed of a microstrip SRR-loaded splitter/combiner configuration etched on a low-loss microwave substrate (Rogers RO3010 with thickness $h=1.27 \mathrm{~mm}$, dielectric constant $\varepsilon_{r}=10.2$ and loss tangent $\tan \delta=0.0023$ ), and two microfluidic channels. The channels are placed on top of the gap region of the SRRs, where the electromagnetic energy is concentrated (Fig. 1). Let us present separately the microwave (microstrip) circuitry and the mechanical parts (including the microfluidic channels).

\section{A. Microwave Circuitry}

The proposed topology of the microwave circuitry (splitter/combiner and SRRs) is depicted in Fig. 1(a), where the relevant dimensions are indicated. By means of this configuration, real time differential measurements can be carried out. By real time we mean that the differential measurement does not need independent measurement of the reference sample and the SUT. The impedance of the parallel transmission lines is $Z_{02}=50 \Omega$. Thus, in order to optimize the matching with the reference impedance $(50 \Omega)$ of the ports, 
impedance inverters implemented by means of quarter wavelength transmission lines with impedance $Z_{01}=35.35 \Omega$ are cascaded between the T-junctions and the ports. The SRRs have been designed in order to exhibit their fundamental resonance in the vicinity of $f_{0}=1 \mathrm{GHz}$ and a deep notch (note that there is some flexibility in the value of $f_{0}$ ). The dimensions of the SRRs and the other geometrical parameters are given in the caption of Fig. 1.

The proposed equivalent circuit model of the SRR-loaded splitter/combiner is depicted in Fig. 2(a) [49],[50]. The SRRs are modeled by means of resonant tanks $L_{s}-C_{s}$ magnetically coupled to the parallel microstrip line sections through mutual inductances $M$. SRR losses are taken into account through the resistance $R_{S}$ (note that substrate and metal losses are not considered since they are small in comparison to SRR losses). The line sections in close proximity to the SRRs are accounted for by the inductance $L$ and the capacitance $C$. Finally, the impedance inverters and the transmission line sections between the T-junctions and the SRRs are modeled by distributed components with the indicated characteristic impedance and electrical length.

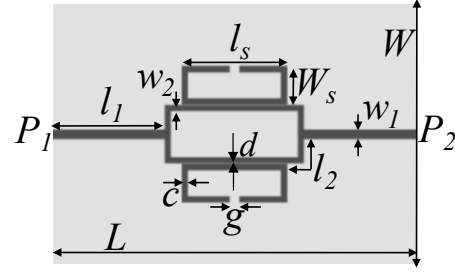

(a)

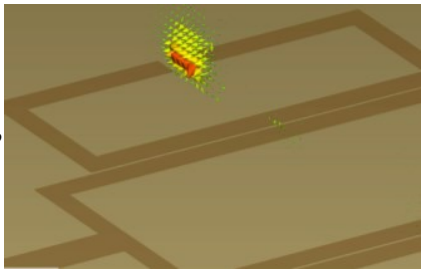

(b)
Fig. 1. (a) Topology of the splitter/combiner configuration and relevant dimensions; (b) electric field distribution at SRR resonance in the vertical plane orthogonal to the axis of the structure crossing the SRR gaps. $L=86$ $\mathrm{mm}, W=62 \mathrm{~mm}$, inverter dimensions are $l_{1}=27 \mathrm{~mm}$ and $w_{1}=2.22 \mathrm{~mm}$, SRR dimensions are $l_{s}=25 \mathrm{~mm}, W_{s}=9 \mathrm{~mm}, c=1.4 \mathrm{~mm}, g=2.4 \mathrm{~mm}$, the slot separation between the lines and the SRRs is $d=0.2 \mathrm{~mm}$, and the dimensions of the transmission lines sections between the T-junctions and the SRRs are $l_{2}$ $=9.21 \mathrm{~mm}, w_{2}=1.34 \mathrm{~mm}$. In (a), the ground plane is depicted in grey.

The circuit of Fig. 2(a) can be transformed to the circuit of Fig. 2(b) through formulas given in [1],[2],[49],[50]. Parameter extraction of the lumped elements in Fig. 2(b) has been carried out from the procedure reported in [2],[51] (applied only to each SRRs and transmission line section coupled to it). Typically, parameter extraction is carried out by excluding losses in both the electromagnetic and circuit simulation. Then losses are introduced in the electromagnetic simulation, and $R_{s}^{\prime}$ is inferred by curve fitting. Note that resonator losses should be included in the model since the notch depth, intimately related to SRR losses, is a relevant parameter in the proposed sensor. Indeed, the notch depth is significantly influenced by losses (typically high) in the liquid under test (LUT), which means that such losses must be included in the circuit model for the description of the whole structure (microwave circuitry plus fluidic channels with liquid inside), as will be discussed later.

To validate the circuit model, we have obtained the frequency response of the symmetric circuit of Fig. 1 through electromagnetic simulation by means of the Ansys HFSS electromagnetic solver [Figs. 3(a) and (b)]. The response exhibits a single notch, as expected, at $f_{0}=1.040 \mathrm{GHz}$, where the resonant $\operatorname{tank} L_{s^{-}}{ }^{-}{ }_{s}$, is an open-circuit, i.e.,

$$
f_{0}=\frac{1}{2 \pi \sqrt{L_{s}^{\prime} C_{s}^{\prime}}}
$$

For the symmetric case (identical resonators) SRR reactive elements satisfy $L_{s 1}^{\prime}=L_{s 2}^{\prime}=L_{s}^{\prime}$ and $C_{s I}^{\prime}=C_{s 2}^{\prime}=C_{s}^{\prime}$. The circuit simulation (inferred by means of Keysight ADS) with the extracted parameters is also depicted in Figs. 3(a) and (b). As can be seen, there is very good agreement in both the magnitude and phase response.

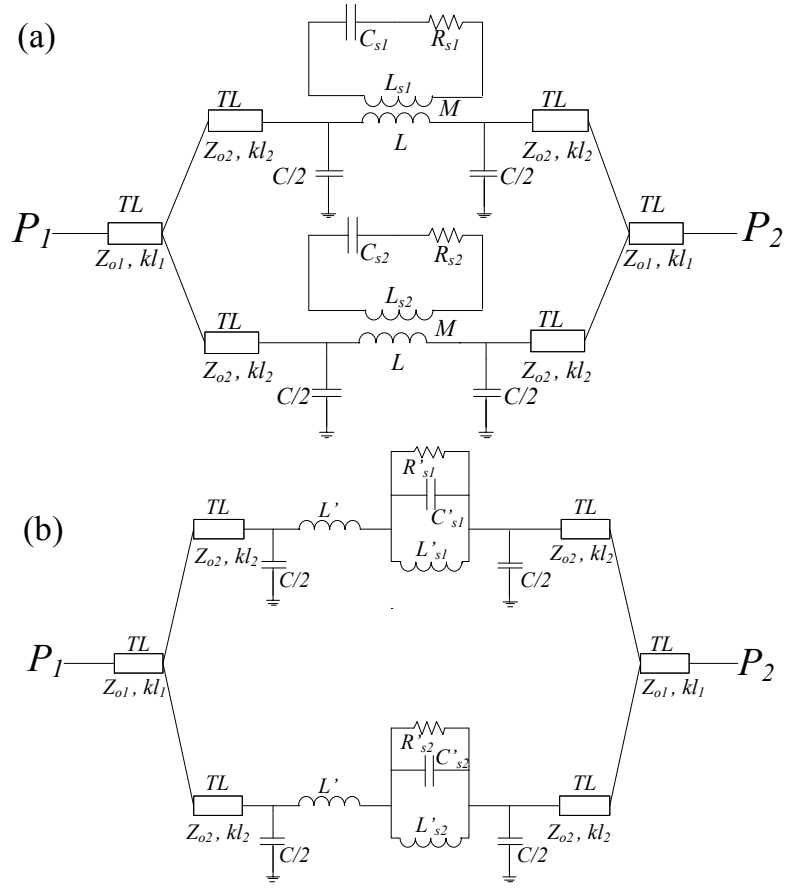

Fig. 2. Circuit model of the topology of Fig. 1, including lumped and distributed components (a), and transformed model (b).
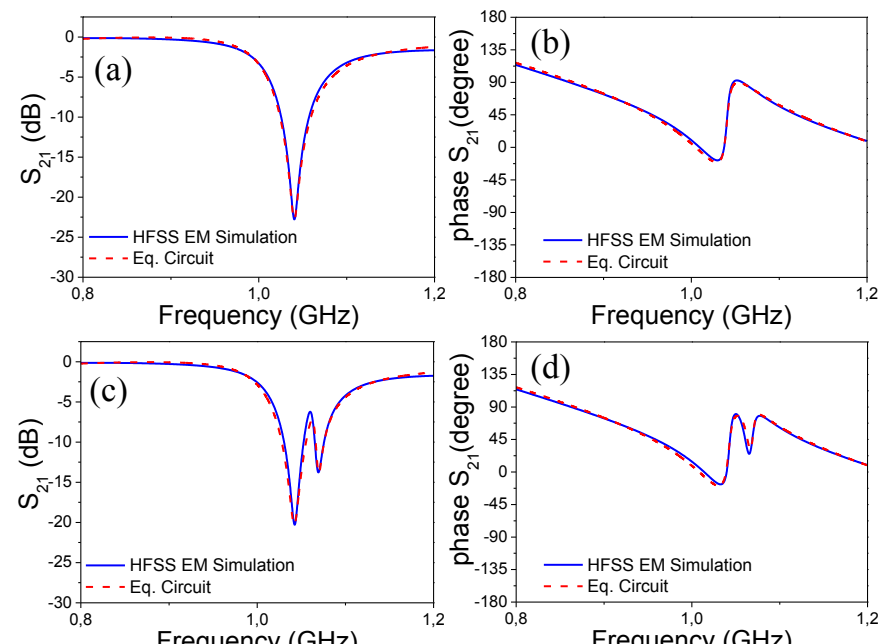

Fig. 3. Magnitude and phase of the transmission coefficient in symmetric (a-b) and asymmetric cases (c-d) for topology of Fig. 1. The element values (in reference to Fig.2 (b) considering symmetry case) are: $L^{\prime}=6.405 \mathrm{nH}$, $C=6.744 \mathrm{pF}, \quad L_{s}^{\prime}=0.585 \mathrm{nH}, \quad C_{s}^{\prime}=40 \mathrm{pF}, \quad R_{s}^{\prime}=510 \Omega, \quad Z_{01}=35.35 \Omega$, $Z_{02}=50 \Omega$, and $k l_{1}=90^{\circ}$, and $k l_{2}=38^{\circ}$. The element values (in reference to Fig.2 (b) considering asymmetry case) are: $C_{s 2}^{\prime}=38.7 \mathrm{pF}$, and $R_{s 2}^{\prime}=676 \Omega$. The other elements remain unchanged. 
We have also considered the structure that is obtained by varying the gap dimensions of the lower SRR (i.e., $g=3.6 \mathrm{~mm}$ for such SRR), providing axial asymmetry. In this case, a pair of transmission zeros appears [Fig. 3(c) and (d)]. The transmission coefficient (magnitude and phase) obtained from circuit simulation is also depicted in the figures, and there is also very good agreement between the simulations in both domains (circuit and electromagnetic). Note that in the circuit simulations of Figs. 3(c) and (d), only the parameters relative to the lower SRR have changed. With these results, the circuit model is validated.

\section{B. Mechanical parts and microfluidic channels}

The mechanical and fluidic parts are located on top of the SRR gap region, where the electromagnetic energy is concentrated [Fig. 1b]. Note that the electric field intensity is significantly larger in the SRR gaps, as compared to the field intensity in the gaps between the SRRs and the lines (splitter /combiner). Figure 4 shows the lateral and top views of the mechanical and fluidic parts of the sensor, including relevant dimensions. Channel dimensions are designated by $h_{c h}, w_{c h}$, and $l_{c h}$. The mechanical parts consist of a Polyether ether ketone (PEEK) structure, designed in order to accommodate the fluidic connectors for liquid injection in a controllable way through a syringe (the reference liquid in channel 1 and the LUT in channel 2). Polydimethylsiloxane (PDMS) has been used to fabricate the fluidic channels due to their biocompatibility and easy fabrication process.
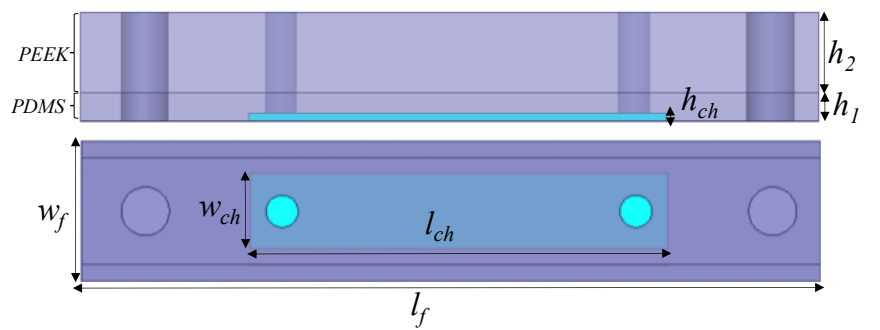

Fig. 4. Lateral (a) and top (b) views of mechanical and fluidic parts of the microwave sensor and relevant dimensions. $h_{c h}=1.5 \mathrm{~mm}, l_{c h}=26 \mathrm{~mm}$, $w_{c h}=4.6 \mathrm{~mm}, l_{f}=46 \mathrm{~mm}, w_{f}=12.6 \mathrm{~mm}, h_{1}=3 \mathrm{~mm}$, and $h_{2}=9 \mathrm{~mm}$.

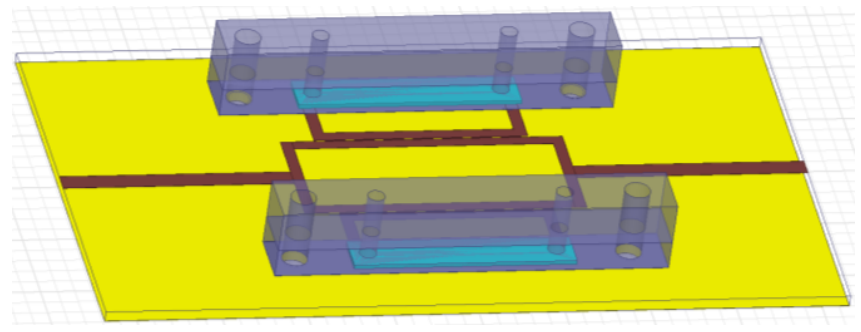

Fig. 5. HFSS 3D model of the proposed sensor.

The 3D view of the whole sensor used for electromagnetic simulation by means of Ansys HFSS is depicted in Fig. 5. The presence of the mechanical and fluidic parts on top of the microwave substrate modifies the element values of the circuit of Fig. 2(b). The response of the whole sensor (with air in the channels), including the measurement, electromagnetic simulation and circuit simulation is depicted in Fig. 6. The good agreement not only validates the circuit model, but also the electrical definition of the different materials in the HFSS electromagnetic simulator.
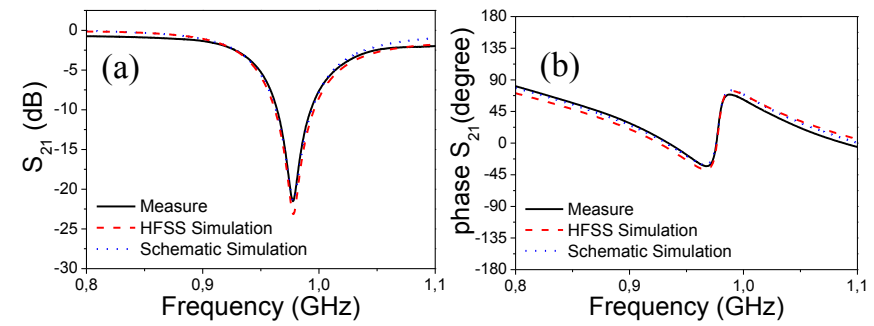

Fig. 6. Measurement, electromagnetic simulation and circuit simulation of the transmission coefficient [magnitude (a) and phase shift (b)] of the proposed sensor with air inside the channels. The extracted element values are: $L^{\prime}=7.405 \mathrm{nH}, \quad C=6.744 \mathrm{pF}, L_{s}^{\prime}=0.697 \mathrm{nH}, \quad C_{s}^{\prime}=38 \mathrm{pF}, \quad R_{s}^{\prime}=518.8 \Omega$, $Z_{01}=35.35 \Omega, Z_{02}=50 \Omega$, and $k l_{1}=90^{\circ}$, and $k l_{2}=38^{\circ}$.

\section{SENSOR FABRICATION AND CALIBRATION}

The microwave circuitry has been fabricated by means of a milling machine (LPKF Protomat H100). Substrate parameters are given at the beginning of Section II, and the thickness of the metal $(\mathrm{Cu})$ layer is $35 \mu \mathrm{m}$. The PDMS channels have been fabricated by means of a mold using a relation between the PDMS and reagent of 10/1. After applying vacuum conditions in order to eliminate all bubbles (that can produce a wrong functionality or degrade the elastic properties of PDMS), the PDMS mold has been introduced in an oven at $60^{\circ} \mathrm{C}$ for 1 hour. The mechanical parts, to accommodate the fluidic connectors and the capillaries (with external and internal diameter of $1.6 \mathrm{~mm}$ and $0.16 \mathrm{~mm}$ respectively), have been fabricated using standard milling machine ( 3 axis CNC $1830 \mathrm{MB}$ ). Finally, all parts have been fixed to the substrate by means of stainless steel screws with $3 \mathrm{~mm}$ diameter. The position for the screws has been optimized (through HFSS simulation) in order to avoid their influence on the electromagnetic fields in the channel and SRR regions. Figure 7 shows the final prototype of the proposed microwave microfluidic sensor, including the $50 \Omega$ connectors soldered at the input and output ports. Due to substrate absorption (modifying substrate permittivity and hence inducing systematic errors in the sensor measurements), a thin film $(0.12 \mathrm{~mm})$ of glass $\left(\varepsilon_{r}=5.5\right)$ has been placed between the microwave substrate and the channels. By this means, absorption is completely avoided, although sensitivity is degraded by the presence of the glass layer. Nevertheless, such separation layer is necessary to prevent direct contact between the liquids and the substrate.

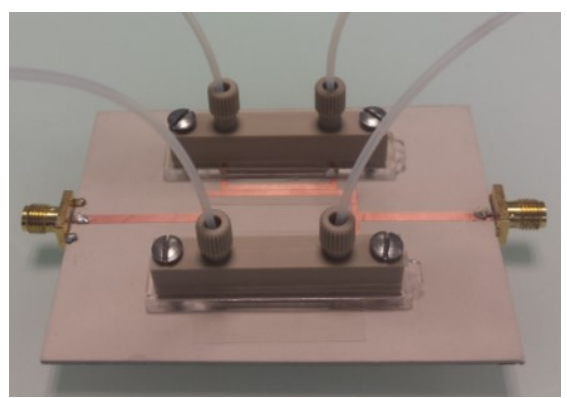

Fig. 7. Photograph of the splitter/combiner microfluidic sensor. 
The complete experimental setup for the calibration and characterization of the sensor is shown in Fig. 8. The measured responses have been obtained by means of the portable Keysight N9923A Fieldfox $R F$ vector network analyzer (VNA). The sensing measurements for calibration have been made with pure DI water, ethanol and DI water with $10 \%$ of ethanol (with well known complex permittivity) since mixtures of DI water and ethanol have been considered later to validate the sensor functionality.

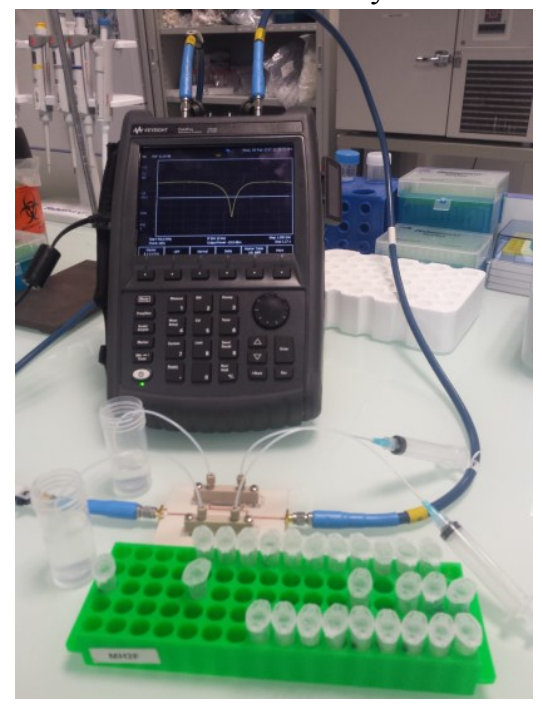

Fig. 8. Experimental setup.

The calibration measurements (Fig. 9) confirm that when both channels are loaded with the same liquid or air (symmetric configuration), the transmission coefficient exhibits only one notch, as expected. If symmetry is disrupted by injecting different liquids in the channels, frequency splitting occurs, resulting in two transmission zeros (obscured by liquid losses). If the LUT is different than the reference liquid (DI water in this paper), the frequency separation, $\Delta f_{z}$, and the difference in the notch depths, $\Delta\left|S_{21}\right|$, should be indicative of the difference in the complex permittivities. By assuming a linear dependence of the complex permittivity with $\Delta f_{z}$ and $\Delta\left|S_{21}\right|$, we can express the variation of the real and imaginary parts of the complex permittivity as [15]

$$
\begin{aligned}
& \Delta \varepsilon^{\prime}=k_{11} \Delta f_{z}+k_{12} \Delta\left|S_{21}\right| \\
& \Delta \varepsilon^{\prime \prime}=k_{21} \Delta f_{z}+k_{22} \Delta\left|S_{21}\right|
\end{aligned}
$$

where $\Delta \varepsilon^{\prime}=\varepsilon^{\prime}{ }_{L U T}-\varepsilon^{\prime}{ }_{r e f}, \Delta \varepsilon \varepsilon^{\prime \prime}=\varepsilon^{\prime \prime}{ }_{L U T}-\varepsilon{ }^{\prime}{ }^{\prime}{ }_{r e f}, \Delta f_{z}=f_{\text {zLUT }}-f_{\text {zref }}$, and $\Delta\left|S_{21}\right|=\left|S_{21}\right|_{L U T}-\left|S_{21}\right|_{\text {ref. }}$. Since the variation in the real and imaginary parts of the complex permittivity of ethanol and $10 \%$ of Ethanol in DI water (as compared to pure DI water) is known, and $\Delta f_{z}$ and $\Delta\left|S_{21}\right|$ can be extracted by measuring the transmission coefficient of the sensor, the unknown variables $\left(k_{11}, k_{12}, k_{21}\right.$, and $\left.k_{22}\right)$ can be calculated. From Fig. 9, these values are found to be $k_{11}=-0.944 \mathrm{MHz}^{-1}, k_{12}=-0.545 \mathrm{~dB}^{-1}$, $k_{21}=0.127 \mathrm{MHz}^{-1}$, and $k_{22}=0.260 \mathrm{~dB}^{-1}$. Note that in (2), the left hand terms actually correspond to difference of the real and imaginary parts of the complex dielectric constant, being thus dimensionless.
Let us now consider the model of Fig. 2(b) with the presence of liquids inside the channels. The real part of the permittivity is related to the density of electromagnetic energy stored inside the liquid and can be modeled by a capacitor $\left(C_{c h}\right)$. The imaginary part is a measure of how dissipative the liquid is (it gives an idea of the attenuation of electromagnetic waves propagating in the fluid). Hence, it can be modeled by a resistor $\left(R_{c h}\right)$. According to these words, in order to account for the presence of fluids in the channels, the model of Fig. 2 must simply include an additional resistor and capacitor, modeling the liquid, as indicated in Fig. 10.

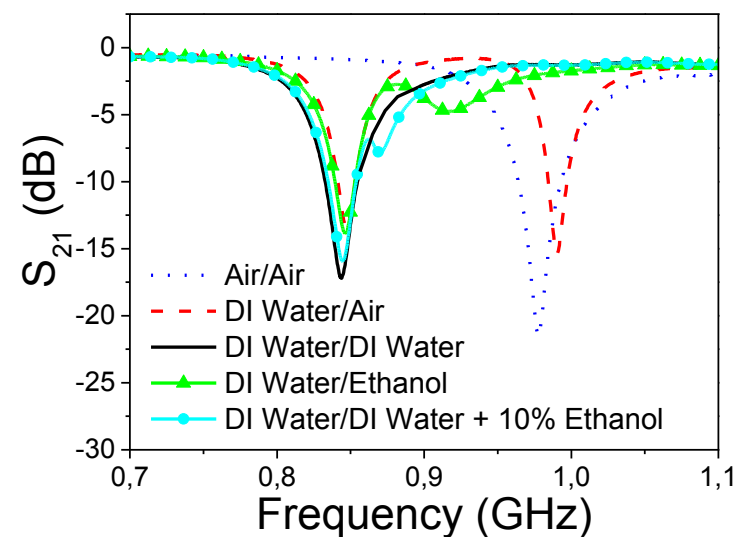

Fig. 9. Measured transmission coefficient for different configurations of channel loading (indicated) to calibrate the sensor. All measurements have been made at room temperature $\left(25^{\circ}\right)$.

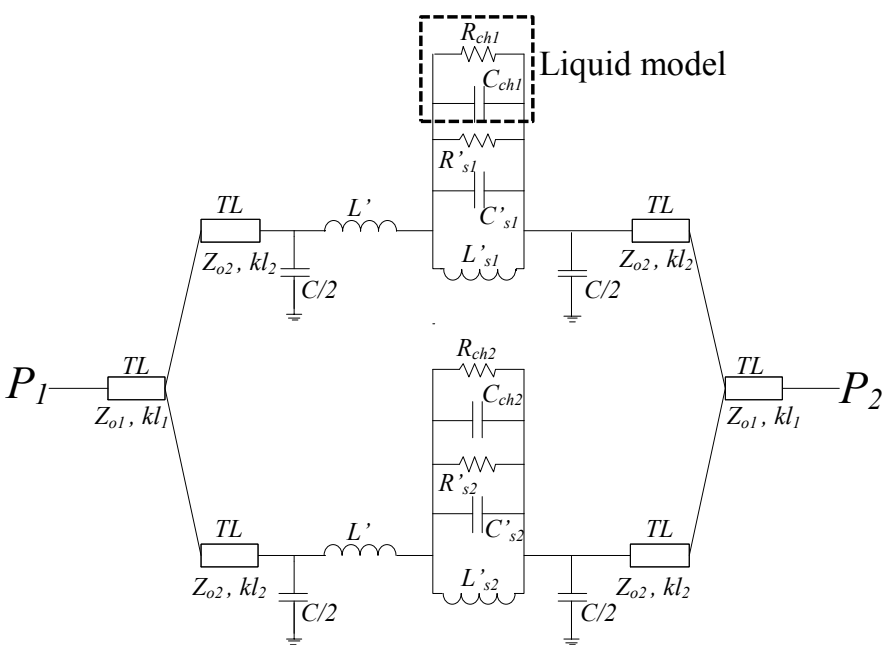

Fig. 10. Equivalent circuit taking into account the presence of liquids inside the channels.

The model is validated by comparing the measured transmission coefficient (magnitude and phase) of various combinations of air and liquids in the channels with the circuit simulations. These comparisons are depicted in Fig. 11. The circuit parameters, excluding those corresponding to the liquid model, are given in the caption of Fig. 6. For coherence, such parameters must be the same regardless of the channel content. Thus, only the parameters modeling the channel content have been adjusted. The capacitance has been determined by the frequency position of the notches, whereas the resistance has been adjusted by the notch depth. These parameters are given in Table I. It is worth mentioning that for 
the air/DI water combination, a finite capacitance and resistance for the air model have been obtained, but the resulting capacitance is very small and the resulting resistance is very high, so that these values are within the tolerance limits of the parameter extraction method. Note that the parameters modeling the DI water are identical for all considered combinations involving such component.
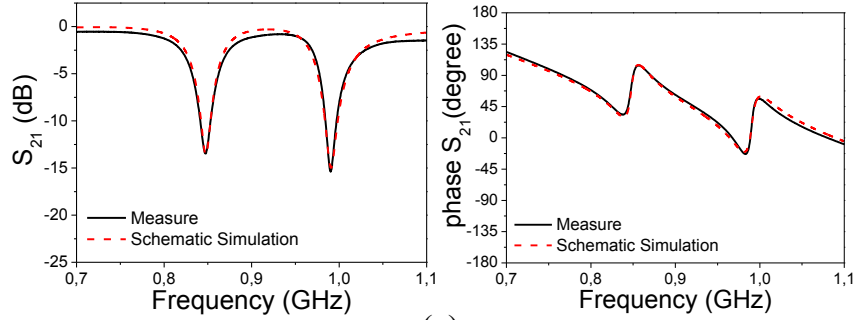

(a)
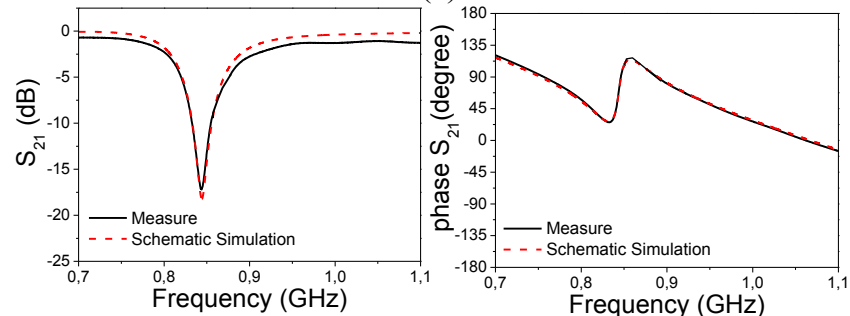

(b)
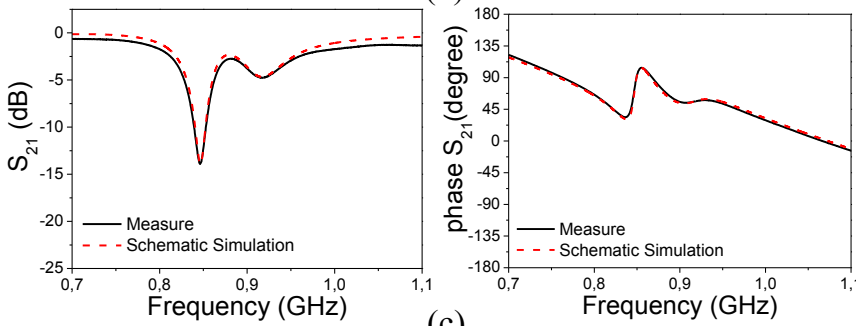

(c)
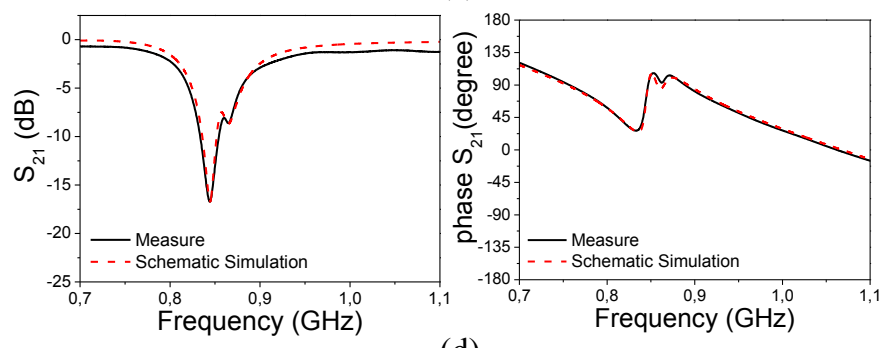

(d)

Fig. 11. Magnitude and phase of the transmission coefficient for various combinations of channel content. (a) Air/DI water, (b) DI water/DI water, (c) DI water/ethanol, and (d) DI water/DI water $+10 \%$ ethanol.

TABLE I. EXTRACTED ELEMENT PARAMETERS OF THE FLUID MODEL FOR CHANNELS 1 AND 2

\begin{tabular}{|c|c|c|c|c|c|}
\hline $\begin{array}{c}\text { Channel } \\
1\end{array}$ & $\begin{array}{l}C_{c h l} \\
(\mathbf{p F})\end{array}$ & $\begin{array}{l}R_{c h l} \\
(\Omega)\end{array}$ & Channel 2 & $\begin{array}{l}C_{c h 2} \\
(\mathbf{p F})\end{array}$ & $\begin{array}{l}R_{c h 2} \\
(\Omega)\end{array}$ \\
\hline DI water & 13 & 1000 & Air & 0.1 & 2930 \\
\hline DI water & 13 & 1000 & DI water & 13 & 1000 \\
\hline DI water & 13 & 1000 & Ethanol & 6.7 & 80 \\
\hline DI water & 13 & 1000 & $\begin{array}{c}\text { DI } \\
\text { water }+10 \% \text { Ethanol }\end{array}$ & 12.2 & 300 \\
\hline
\end{tabular}

\section{SENSOR VALIDATION AND RESUltS}

To validate the proposed microwave sensor, mixtures of DI water and ethanol, with volume fraction of ethanol varying from $10 \%$ to $100 \%$ by steps of $10 \%$, have been characterized, considering DI water as reference liquid. We have considered DI water as reference liquid since the relative variation of the permittivity of most LUT, as compared to DI water, is small. By these means, the linearity assumption in expressions (2) is reasonable. The measured transmission coefficient for the set of samples is plotted in Fig. 12(a) along with the extracted values of $\Delta f_{z}$ and $\Delta\left|S_{21}\right|$ [Fig. 12(b)]. The difference between the frequency position and magnitude (notch depth) of the two transmission zeros increases as the percentage of ethanol increases. From the knowledge of the complex dielectric constant of DI water at $0.87 \mathrm{GHz}$, i.e., 80.86 - 3.04j (the one of ethanol is $27.86-10 \mathrm{j}$ at such frequency) [52], we have inferred the real and imaginary parts of the complex dielectric constant for the considered DI water/ethanol mixtures (Fig. 13). These data have been obtained through equations 2 (a) and (b). The Weiner model [53] has been used to calculate the static upper (W.U) and lower (W.L) limits of the real and imaginary parts of the complex dielectric constant as a function of the ethanol content (also depicted in Fig. 13). The inferred complex dielectric constants for the different mixtures of DI water and ethanol are between the Weiner limits. Thus, these results validate the functionality of the proposed sensor.

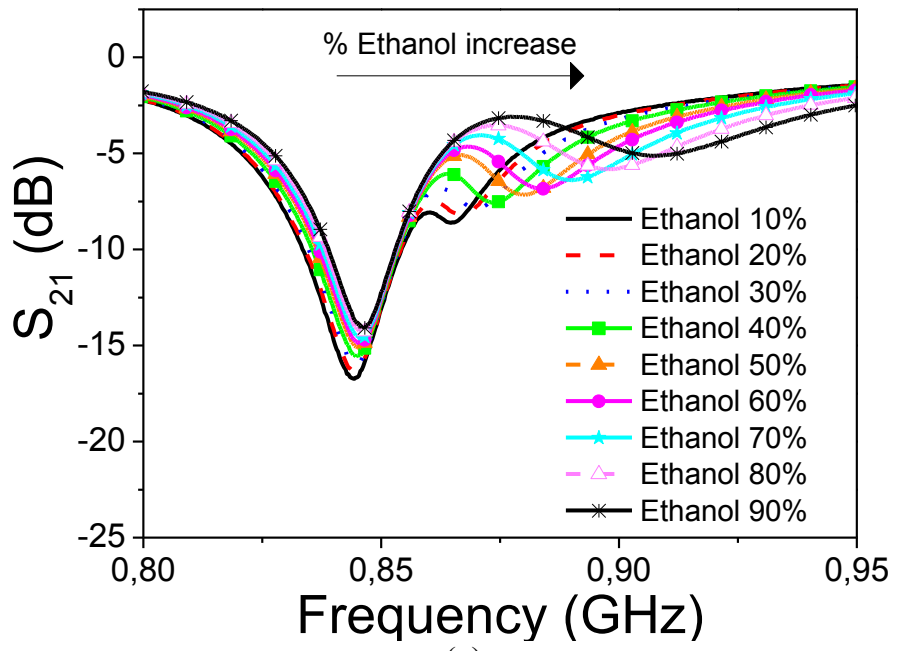

(a)

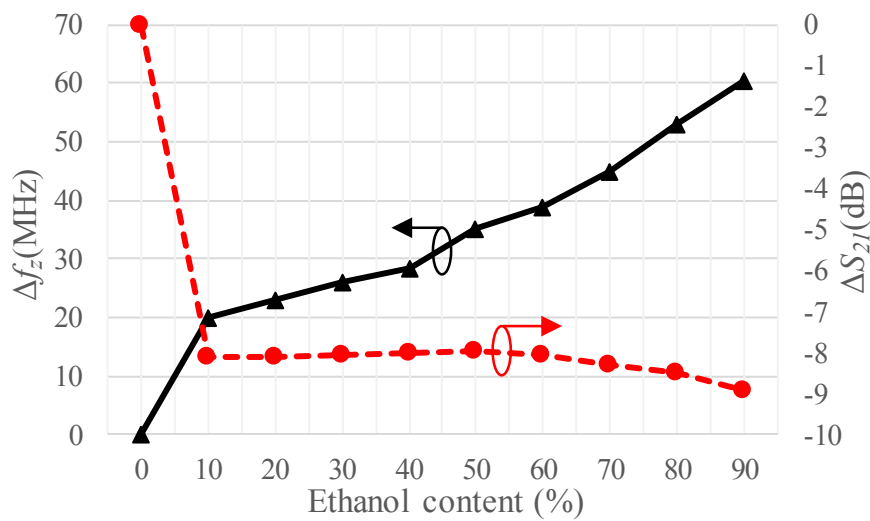

(b)

Fig. 12. Measured transmission coefficient (magnitude) for different mixtures of DI water and ethanol (LUT), considering DI water as reference liquid (a), and representation of $\Delta f_{z}$ and $\Delta\left|S_{21}\right|$ as a function of the ethanol content (b). All measurements have been taken at room temperature $\left(25^{\circ}\right)$ 


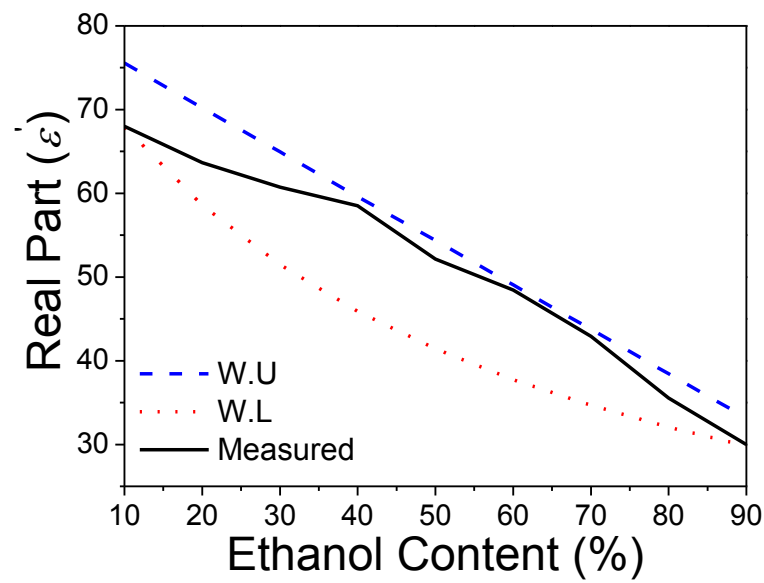

(a)

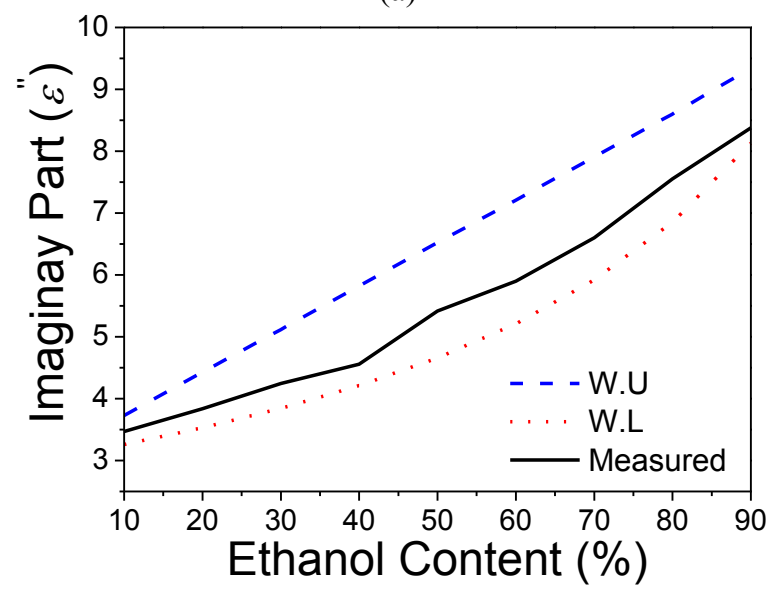

(b)

Fig. 13. Extracted value for the real (a) and imaginary (b) parts of the complex dielectric constant in mixtures of DI water/ethanol. The static Weiner model (upper and lower limits) is also included for comparison purposes.

\section{DISCUSSION AND COMPARISON TO OTHER FLUIDIC SENSORS}

In the introductory section, it was pointed out that in order to optimize sensitivity at small perturbations, it is necessary to set the length of the transmission line sections between the Tjunctions and the SRRs, $l_{2}$, to a certain value (optimum length). Such length is the one that provides a short circuit at the T-junction planes at the resonance frequency of the reference resonator. Obviously, if the reference resonator is loaded with a reference liquid, the SRR resonance frequency corresponding to such liquid loading is the one that should be considered. Let us assume that such angular frequency is $\omega_{0}$. At this frequency, the series branch, composed by the parallel resonant tank plus the series inductance $L^{\prime}$ [see Fig. 2(b)] opens (for the sake of the present calculation SRR losses are neglected). Thus, in order to calculate the optimum length, it is necessary to force the impedance seen at the T-junction plane to zero, by considering that the load at the opposite extreme of the considered line is simply the shunt capacitance $C / 2$. This gives the optimum length, which has been found to be (in terms of electrical length)

$$
\theta_{o p t}=k l_{2, o p t}=\arctan \left(\frac{2}{C \omega_{0} Z_{o 2}}\right)
$$

If the reference liquid is well specified, the optimum length should be considered in order to optimize sensitivity at small perturbations. However, such optimum length is not compatible with versatility, i.e., the capability of the sensor to operate by considering different reference liquids (providing different values of $\omega_{0}$ ). For this main reason, we do not have optimized $l_{2}$. By contrast, we have considered a short (arbitrary) length in order to reduce sensor dimensions. Despite this fact, the sensor functionality has been demonstrated, as derived from the minimum detectable volume fraction of ethanol $(10 \%)$. This was the main aim of the paper, rather than optimizing sensitivity. Nevertheless, for a specific reference liquid, sensor sensitivity at small perturbations can be improved by setting the length $l_{2}$ to the value resulting from (3). It is interesting to mention that the fact the $l_{2}$ is not set to the optimum value, for the given reasons, explains the slight variation of the first resonance frequency in Fig. 12(a). As reported in detail in [47], if $l_{2}$ is not set to the optimum value, the two resonance frequencies resulting with an asymmetric loading are not the resonance frequencies of the loaded resonators, but are due to an interfering phenomenon, and both frequencies depend on both loadings. Nevertheless, this fact does not prevent from using the proposed system, with $l_{2}$ set to a value below the optimum value (with water as reference liquid), for the measurement of the complex permittivity of liquids, since it is based on the use of calibration curves.

We would like also to mention that a sensing structure topologically similar to the one presented in this paper is reported in [54]. In that paper, a pair of SRRs and a splitter/combiner is also used. However, the sensor uses two branches each one loaded with a resonant element and a series gap, providing a band pass functionality, rather than a stop band (as in our work). In [54], a Wilkinson divider/combiner, providing port isolation, is used. By this means, loading in one branch does not affect the other sensor branch. However, in stop band configuration, the resonance frequencies (notches) are consequence of an interfering phenomenon, as has been pointed out before, and port isolation does not prevent from loading effects between both branches for asymmetric loading.

A comparison to other recent microwave fluidic sensors working with mixtures of DI water/Ethanol is given in Table II. As can be seen, the proposed approach is competitive in terms of the minimum detectable fractional volume $\left(F_{v}\right)$ of ethanol, although sensitivity at small perturbations has not been optimized (for the reasons mentioned before). Concerning the average frequency sensitivity, $S_{a v}$, or variation of the resonance frequency with the dielectric constant over the considered input variable span, it can be seen that the values given in the table are very different. However, the working frequencies $\left(f_{0}\right)$ are also significantly diverse. Therefore, for proper comparison, the fractional variation of frequency should be considered. This later sensitivity, defined as the frequency variation over the average frequency and over the (dimensionless) dielectric constant interval, and designated as $S_{a v, f}$, is also given in Table II. Note that $S_{a v, f}$ is 
dimensionless and it is expressed as percentage in Table II as long as the relative frequency variation is given in $\%$.

In view of the values of $S_{a v, f}$, it can be appreciated that in the works where SRRs are used ([15] and the present one), the sensors exhibit smaller frequency sensitivity as compared to the sensors in [14],[17], where complementary split ring resonators (CSRRs) are used as sensing elements. The reason is that the CSRR exhibits an edge capacitance along the whole perimeter of the particle, and hence it is intrinsically more sensitive to the effects of a dielectric load (e.g. a liquid) on top of it. Nevertheless, it is worth mentioning that the sensitivity in the notch depth, related to losses, is comparable or even worst when CSRRs are used.

In the works [12],[16], a resonant element exhibiting a significant edge capacitance, etched in the central strip of a coplanar waveguide (CPW), has been considered, and the frequency sensitivity is very competitive. In the present work, rather than optimizing sensitivity, the main aim has been to demonstrate the viability and functionality of differential mode microfluidic sensors based on frequency splitting, where real-time measurements and robustness in front of environmental changes are key aspects.

TABLE II

COMPARISON OF VARIOUS DI WATER/ETHANOL MICROWAVE MICROFLUIDIC SENSORS

\begin{tabular}{c|c|c|c|c|c} 
Reference & $\boldsymbol{f}_{\mathbf{0}}(\mathbf{G H z})$ & $\boldsymbol{S}_{\boldsymbol{a v}}(\mathbf{M H z})$ & $\boldsymbol{S}_{\boldsymbol{a v}, \boldsymbol{f}}(\mathbf{\%})$ & $\boldsymbol{F}_{\boldsymbol{v}}(\mathbf{\%})$ & $\begin{array}{c}\text { Real-time } \\
\text { Measurement }\end{array}$ \\
\hline$[12]$ & 20 & 59.75 & 2.98 & 5 & No \\
\hline$[14]$ & 2 & 4.76 & 2.38 & 10 & No \\
\hline$[15]$ & 1.9 & 1.53 & 0.81 & 10 & No \\
\hline$[17]$ & 3.5 & 9.16 & 2.61 & 10 & No \\
\hline This work & $\mathbf{0 . 8 7}$ & $\mathbf{0 . 7 9}$ & $\mathbf{0 . 9 1}$ & $\mathbf{1 0}$ & Yes \\
\hline
\end{tabular}

\section{CONCLUSIONS}

In this work, differential mode microwave microfluidic sensors based on frequency splitting and implemented by means of a splitter/combiner configuration, have been presented. The sensing elements are two identical split ring resonators (SRRs) etched in close proximity to the parallel transmission line sections of the splitter/combiner. The fluidic channels are placed on top of the gap region of the SRRs, where the electric field intensity is magnified and, hence, it is the most sensitive region for the measurement of complex permittivities in the liquids under test (LUT). In the paper, we have proposed a circuit model that accurately predicts the frequency response of the sensor, including the responses when the channels are loaded with liquids. Such liquids have been accounted for in the model by means of a capacitor (modeling the real part of the complex permittivity) and a resistor (describing liquid losses), both disposed in parallel to the resonant elements modeling the SRRs. We have calibrated the sensor through measurements with different combinations of air, DI water and ethanol in the channels, where the output variables have been the difference in frequency and the difference in the notch magnitude (in $\mathrm{dB}$ ) between the two transmission zeros that are generated under asymmetric loading. Since the complex dielectric constant of such liquids is known, it has been possible to determine the four coefficients of the two equations that link the real and imaginary part of the differential dielectric constant (the one of the LUT with regard to the one of the reference liquid) with the output variables. By considering DI water as reference liquid, we have obtained the complex dielectric constant in solutions of DI water/ethanol as a function of the volume fraction of ethanol. The results obtained have been found to lie within the limits predicted by the Weiner model, and hence these results validate the functionality of the proposed differential mode microfluidic sensor. Despite the fact that sensitivity at small perturbations has not been optimized (in favor of versatility and size reduction), volume fractions of $10 \%$ ethanol, i.e., comparable to reported values in the literature, have been resolved. The average frequency sensitivity of the proposed sensor has been found to be similar to the one found in other (non-differential) sensors based on SRRs, but smaller than the sensitivity obtained in microfluidic sensors based on CSRRs or other resonant elements more sensitive to dielectric loading. However, the differential mode microfluidic sensor concept proposed in this paper, based on a splitter/combiner configuration, can be easily extended to CSRRs and to other (more sensitive) sensing elements. The main contribution of this paper concerns the fact that, with differential mode operation, real-time measurements can be performed since the reference liquid and the LUT are syringed in different channels. Additionally, the sensor is robust in front of cross sensitivities caused by external factors (such as temperature variations, moisture, etc.), since such perturbations are seen as common mode stimulus.

\section{REFERENCES}

[1] R. Marques, F. Martin, and M. Sorolla, Metamaterials with Negative Parameters: Theory, Design and Microwave Applications, John Wiley, Hoboken, NJ, USA, 2008.

[2] F. Martín, Artificial Transmission Lines for RF and Microwave Applications, John Wiley, Hoboken, NJ, USA, 2015.

[3] T. Driscoll, G.O. Andreev, D.N. Basov, S. Palit, S.Y. Cho, N.M. Jokerst, and D.R. Smith, "Tuned permeability in terahertz split ring resonators for devices and sensors," Appl. Phys. Lett., vol 91, paper 062511-1-3, 2007.

[4] R. Melik, E. Unal, N.K. Perkgoz, C. Puttlitz, and H.V. Demir, "Metamaterial-based wíreles strain sensors," Appl. Phys. Lett., vol. 95, paper 011106-1-3, 2009.

[5] R.A. Yogi, R.S. Parolia, R.N. Karekar, and R.C. Aiyer, "Microwave microstrip ring resonador as a paper moisture sensor: study with different grammage," Meas. Sci. Technol. vol 13, pp. 1558-1562, 2002.

[6] X-J. He, Y. Wang, J-M. Wang, and T-L. Gui, "Thin film sensor based tip-shaped splits ring resonator metamaterial for microwave application," Microsystems Technology, vol. 16, pp. 1735-1739, 2010.

[7] M. S. Boybay and O. M. Ramahi, "Material characterization using complementary split-ring resonators," IEEE Trans. Instrum. Meas., vol. 61, no. 11, pp. 3039-3046, Nov. 2012.

[8] C.-S. Lee and C.-L. Yang, "Complementary split-ring resonators for measuring dielectric constants and loss tangents," IEEE Microw. Wireless Compon. Lett., vol. 24, no. 8, pp. 563-565, Aug. 2014.

[9] C.-L. Yang, C.-S. Lee, K.-W. Chen, and K.-Z. Chen, "Noncontact measurement of complex permittivity and thickness by using planar resonators," IEEE Trans. Microw. Theory Techn., vol. 64, no.1, pp. 247-257, Jan. 2016.

[10] M. Puentes, C. Weiß, M. Schüßler, and R. Jakoby, "Sensor array based on split ring resonators for analysis of organic tissues," in IEEE MTT-S Int. Microw. Symp., Baltimore, MD, USA, Jun. 2011, pp. 1-4.

[11] M. Puentes, Planar Metamaterial Based Microwave Sensor Arrays for Biomedical Analysis and Treatment. Springer, Heidelberg, Germany, 2014 
[12] T. Chretiennot, D. Dubuc, and K. Grenier, "A microwave and microfluidic planar resonator for efficient and accurate complex permittivity characterization of aqueous solutions," IEEE Trans. Microw. Theory Techn., vol. 61, no. 2, pp. 972-978, Feb. 2013.

[13] A. Abduljabar, D. Rowe, A. Porch, and D. Barrow, "Novel microwave microfluidic sensor using a microstrip split-ring resonator," IEEE Trans. Microw. Thory Techn., vol. 62, no. 3, pp. 679-688, Mar. 2014.

[14] A. Ebrahimi, W. Withayachumnankul, S. Al-Sarawi, D. Abbott, "Highsensitivity metamaterial-inspired sensor for microfluidic dielectric characterization," IEEE Sensors J., vol. 14, no. 5, pp. 1345-1351, May 2014

[15] W. Withayachumnankul, K. Jaruwongrungsee, A. Tuantranont, C. Fumeaux, and D. Abbott, "Metamaterial-based microfluidic sensor for dielectric characterization," Sensor Actuat. A Phys., vol. 189, pp. 233-237, Jan. 2013.

[16] T. Chretiennot, D. Dubuc, and K. Grenier, "Optimized Electromagnetic Interaction Microwave Resonator/Microfluidic Channel for Enhanced Liquid Bio-Sensor," in Europ. Microw. Conf., pp. 464-467, Dec. 2013.

[17] A. Salim and S. Lim, "Complementary Split-Ring Resonator-Loaded Microfluidic Ethanol Chemical Sensor," Sensors, vol. 16, pp. 1-13, 2016.

[18] H.-J. Lee and J.-G. Yook, "Biosensing using split-ring resonators at microwave regime," App. Phys. Lett., vol. 92, no. 25, p. 254103, 2008.

[19] E. Ekmekci and G. Turhan-Sayan, "Multi-functional metamaterial sensor based on a broad-side coupled SRR topology with a multi-layer substrate," App. Phys. A , vol. 110, no. 1, pp. 189-197, Jan. 2013.

[20] C. Damm, M. Schussler, M. Puentes, H. Maune, M. Maasch, and R. Jakoby, "Artificial transmission lines for high sensitive microwave sensors," IEEE Sensors Conf., Christchurch, New Zealand, pp. 755-758, Oct. 2009.

[21] C. Damm, Artificial Transmission Line Structures for Tunable Microwave Components and Microwave Sensors, Shaker Verlag, Aachen, Germany, 2011.

[22] M. Schueler, C. Mandel, M. Puentes, and R. Jakoby, "Metamaterial inspired microwave sensors," IEEE Microw. Mag., vol. 13, no. 2, pp. 57-68, Mar. 2012

[23] T. Chen, S. Li, and H. Sun, "Metamaterials application in sensing", Sensors, vol. 12, pp. 2742-2765, June 2012.

[24] L. Su J. Mata-Contreras, P. Vélez and F. Martín, "Estimation of conductive losses in complementary split ring resonator (CSRR) loading an embedded microstrip line and applications", IEEE MTT-S Int. Microw. Symp. (IMS'17), Honolulu, Hawaii, June 2017.

[25] M. Tiuri, "Microwave Sensor Applications in Industry," in Europ. Microw. Conf., pp. 25-32, Sep. 1987.

[26] J. G. Webster, The Measurement Instrumentation and Sensors Handbook. Boca Raton, FL, USA: CRC, 1999.

[27] J. Fraden, Handbook of Modern Sensors: Physics, Design, and Applications, 3rd ed. New York, NY, USA, Springer, 2004.

[28] J. Naqui, M. Durán-Sindreu and F. Martín, "Novel sensors based on the symmetry properties of split ring resonators (SRRs)," Sensors, vol 11, pp. 7545-7553, 2011.

[29] J. Naqui, Symmetry properties in transmission lines loaded with electrically small resonators: circuit modeling and applications, Springer, Heidelberg, Germany, 2016.

[30] J. Naqui, M. Durán-Sindreu, and F. Martín, "Alignment and Position Sensors Based on Split Ring Resonators," Sensors, vol. 12, pp. 11790-11797, 2012.

[31] A.K. Horestani, C. Fumeaux, S.F. Al-Sarawi, and D. Abbott, "Displacement sensor based on diamond-shaped tapered split ring resonator," IEEE Sens. J., vol. 13, pp. 1153-1160, 2013.

[32] A.K. Horestani, D. Abbott, and C. Fumeaux, "Rotation sensor based on horn-shaped split ring resonator," IEEE Sens. J., vol. 13, pp. 3014-3015, 2013.

[33] J. Naqui and F. Martín, "Transmission Lines Loaded with Bisymmetric Resonators and Their Application to Angular Displacement and Velocity Sensors," IEEE Trans. Microw. Theory Techn., vol. 61, no. 12, pp. 4700-4713, Dec. 2013

[34] J. Naqui and F. Martín, "Angular displacement and velocity sensors based on electric-LC (ELC) loaded microstrip lines," IEEE Sensors J., vol. 14, no. 4, pp. 939-940, Apr. 2014.

[35] A.K. Horestani, J. Naqui, D. Abbott, C. Fumeaux, and F. Martín, "Twodimensional displacement and alignment sensor based on reflection coefficients of open microstrip lines loaded with split ring resonators," Elec. Lett., vol. 50, pp. 620-622, Apr. 2014.
[36] J. Naqui and F. Martín, "Microwave sensors based on symmetry properties of resonator-loaded transmission lines: a review," Journal of Sensors, vol. 2015, Article ID 741853, 10 pages, 2015.

[37] J. Naqui, J. Coromina, A. Karami-Horestani, C. Fumeaux, and F. Martín, "Angular displacement and velocity sensors based on coplanar waveguides (CPWs) loaded with S-shaped split ring resonator (S-SRR)," Sensors, vol. 15, pp. 9628-9650, 2015.

[38] J. Naqui, F. Martín, "Application of broadside-coupled split ring resonator (BC-SRR) loaded transmission lines to the design of rotary encoders for space applications", IEEE MTT-S Int. Microw. Symp. (IMS'16), San Francisco, May 2016.

[39] J. Mata-Contreras, C. Herrojo, and F. Martín, "Application of split ring resonator (SRR) loaded transmission lines to the design of angular displacement and velocity sensors for space applications", IEEE Trans. Microw. Theory Techn., submitted.

[40] A. K. Horestani, J. Naqui, Z. Shaterian, D. Abbott, C. Fumeaux, and F. Martín, "Two-Dimensional Alignment and Displacement Sensor based on Movable Broadside-coupled Split Ring Resonators," Sensors and Actuators A, vol. 210, pp. 18-24, April 2014

[41] J. Naqui, C. Damm, A. Wiens, R. Jakoby, L. Su, and F. Martín, "Transmission lines loaded with pairs of magnetically coupled stepped impedance resonators (SIRs): modeling and application to microwave sensors," IEEE MTT-S Int. Microwave Symp., Tampa, FL, USA, June 2014, pp. 1-4.

[42] L. Su, J. Naqui, J. Mata-Contreras, and F. Martín "Modeling metamaterial transmission lines loaded with pairs of coupled split ring resonators," IEEE Ant. Wireless Propag. Lett., vol. 14, pp. 68-71, 2015.

[43] L. Su, J. Naqui, J. Mata, F. Martín, "Dual-band epsilon-negative (ENG) transmission line metamaterials based on microstrip lines loaded with pairs of coupled complementary split ring resonators (CSRRs): modeling, analysis and applications", $9^{\text {th }}$ International Congress on Advanced Electromagnetic Materials in Microwaves and Optics, Metamaterials 2015, Oxford, UK, Sep., 7-12, 2015.

[44] L. Su, J. Naqui, J. Mata-Contreras, P. Vélez, F. Martín, “Transmission line metamaterials based on pairs of coupled split ring resonators (SRRs) and complementary split ring resonators (CSRR): a comparison to the light of the lumped element equivalent circuits", International Conference on Electromagnetics for Advanced Applications, ICEAA 2015, Torino, Italy, 7-11 Sep. 2015.

[45] L. Su, J. Naqui, J. Mata-Contreras, and F. Martín, "Modeling and applications of metamaterial transmission lines loaded with pairs of coupled complementary split ring resonators (CSRRs)," IEEE Ant. Wireless Propag. Lett., vol. 15, pp. 154-157, 2016.

[46] J. Naqui, C. Damm, A. Wiens, R. Jakoby, L. Su, J. Mata-Contreras, and F. Martín, "Transmission Lines Loaded with Pairs of Stepped Impedance Resonators: Modeling and Application to Differential Permittivity Measurements", IEEE Trans. Microw. Theory Techn., vol. 64, no. 11, pp. 3864-3877, Nov. 2016.

[47] L. Su, J. Mata-Contreras, J. Naqui, and F. Martín, "Splitter/combiner microstrip sections loaded with pairs of complementary split ring resonators (CSRRs): modeling and optimization for differential sensing applications", IEEE Trans. Microw. Theory Techn., vol. 64(12), pp. 4362-4370, Dec. 2016.

[48] L. Su, J. Mata-Contreras, and F. Martín, "Configurations of splitter/combiner microstrip sections loaded with stepped impedance resonators (SIRs) for sensing applications", Sensors, vol. 16(12), paper $2195,2016$.

[49] F. Martín, F. Falcone, J. Bonache, R. Marqués and M. Sorolla, "Split ring resonator based left handed coplanar waveguide", Appl. Phys. Lett., vol. 83, pp. 4652-4654, Dec. 2003.

[50] F. Aznar, J. Bonache and F. Martín, "Improved circuit model for left handed lines loaded with split ring resonators", Appl. Phys. Lett., vol. 92, paper 043512, Feb. 2008

[51] F. Aznar, M. Gil, J. Bonache, J.D. Baena, L. Jelinek and R. Marqués and F. Martín, "Characterization of miniaturized metamaterial resonators coupled to planar transmission lines," J. Appl. Phys., vol. 104, paper 114501-1-8, Dec. 2008

[52] Hayes, B. L., Microwave Synthesis:Chemistry at the speed of Light, CEM Publishing, Matthews, NY, 2006.

[53] O. Weiner, "Die theorie des Mischkorpers fur das Feld der statonare Stromung i. die mittelwertsatze fur kraft, polarisation und energie", Der Abhandlungen der Mathematisch-Physischen Klasse der Konigl. Sachsischen Gesellschaft der Wissenschaften, vol.32, pp.509-604, 1912.

[54] M.H. Zarifi, S. Farsinezhad, B.D. Wiltshire, M. Abdorrazaghi, N. Mahdi, P. Kar, M. Daneshmand, and K. Shankar, "Effect of phosphonate 
monolayer adsorbate on the microwave photoresponse of $\mathrm{TiO} 2$ nanotube membranes mounted on a planar double ring resonator", Nanotechnology, vol. 27, paper 375201, Sep. 2016.

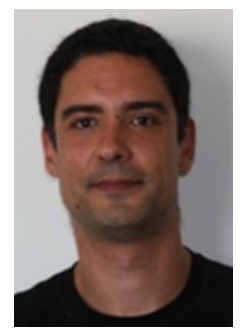

Paris Vélez (S'10-M'14) was born in Barcelona,Spain, in 1982. He received the degree in telecommunications engineering, specializing in electronics, the Electronics Engineering degree, and the Ph.D. degree in electrical engineering from the Universitat Autònoma de Barcelona, Barcelona, in 2008, 2010, and 2014, respectively. His Ph.D. thesis concerned common mode suppression differential microwave circuits based on metamaterial concepts and semilumped resonators. He is currently involved in the subjects related to metamaterials sensors for fluidics detection at LAAS-CNRS through a TECNIOSpring fellowship cofounded by the Mari Curie program. His current research interests include the miniaturization of passive circuits $\mathrm{RF} /$ microwave and sensors-based metamaterials.

Dr. Vélez was the recipient of a predoctoral teaching and research fellowship by the Spanish Government from 2011 to 2014. He is a Reviewer for the IEEE TRANSACTIONS ON MICROWAVE THEORY AND TECHNIQUES and for other journals.

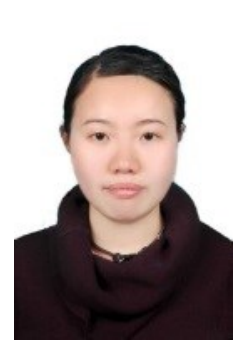

Lijuan Su (S'14) was born in Qianjiang, China, in 1983. She received the bachelor's degree in Communication Engineering and M.E. degree in Circuits and Systems from Wuhan University of Technology, Wuhan, China, in 2005 and 2013, respectively. She is currently pursuing the Ph.D. degree in metamaterials applied to $\mathrm{RF} /$ microwave sensors at the Universitat Autonoma de Barcelona, Barcelona, Spain.

From 2005 to 2009, she was an engineer with China Telecom Corporation Ltd., Hubei, China.

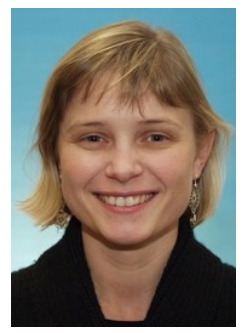

Katia Grenier (S'99, M'03) received her M.S. and $\mathrm{Ph} . \mathrm{D}$. degrees in electrical engineering from the University of Toulouse, Toulouse, France, in 1997 and 2000, respectively. She was engaged in microelectromechanical systems (MEMS) circuits on silicon. She was a Postdoctoral Fellow at Agere Systems (Bell Labs). In 2001, she joined the Laboratory of Analysis and Architecture of System of the National Scientific Research Center (LAAS-CNRS), Toulouse, France. From 2007 to 2009, she was with the Laboratory for Integrated Micromechatronic Systems CNRS (LIMMS-CNRS)/Institute of Industrial Science (IIS), Universtity of Tokyo, Tokyo, Japan, where she was engaged in launching research activities on microwave-based biosensors. Her research interests in LAAS-CNRS are now focused on the development of fluidic-based microsystems, notably for biological and medical applications at the cellular and molecular levels. Dr. Grenier is a member of the IEEE MTT-10 Technical Committee on Biological effect and medical applications of RF and microwave of the IEEE Microwave Theory and Techniques Society.

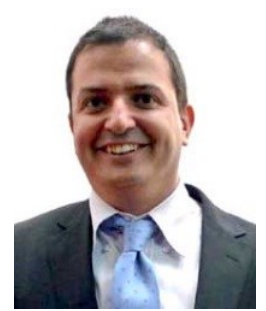

Javier Mata-Contreras was born in 1976 in Málaga (Spain). $\mathrm{He}$ received the Ingeniería de Telecomunicación Degree from the Universidad de Málaga (UMA) in 2000 and the $\mathrm{PhD}$ degree from the same university in 2010, with the Thesis "Distributed Amplifiers and Mixers with Transmission Lines based on Metamaterials". In 2000, he joined the UMA Department of Ingeniería de Comunicaciones UMA as Assistant Professor. He is currently working at CIMITEC and the Universitat Autònoma de Barcelona as Visitant Professor. His research interests include active and passive microwave devices and active distributed circuits based on metamaterials, among others.

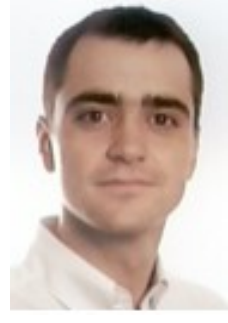

David Dubuc (S'99, M'03) received the Agregation degree from the Ecole Normale Supérieure de Cachan, Paris, France, in 1996, and the M.S. and Ph.D. degrees in electrical engineering from the University of Toulouse, Toulouse, France, in 1997 and 2001, respectively. From 2002 to 2013, he was an Associate Professor with the University of Toulouse, and a Researcher with the Laboratory of Analysis and Architecture of System part of National Scientific Research Center (LAAS-CNRS), Toulouse, France. From 2007 to 2009, he was a Visiting Senior Researcher with the Laboratory of Integrated Micromechatronic Systems (LIMMS-CNRS)/Institute of Industrial Science (IIS), University of Tokyo, Tokyo, Japan. Since 2013, he is Professor with the University of Toulouse. His research interests include the development of microwave circuits integrated due to microtechnologies and their application to wireless telecommunication and biology.

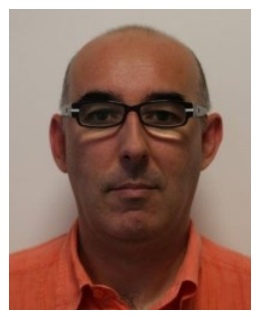

Ferran Martín (M'04-SM'08-F'12) was born in Barakaldo (Vizcaya), Spain in 1965. He received the B.S. Degree in Physics from the Universitat Autònoma de Barcelona (UAB) in 1988 and the $\mathrm{PhD}$ degree in 1992. From 1994 up to 2006 he was Associate Professor in Electronics at the Departament d'Enginyeria Electrònica (Universitat Autònoma de Barcelona), and since 2007 he is Full Professor of Electronics. In recent years, he has been involved in different research activities including modelling and simulation of electron devices for high frequency applications, millimeter wave and $\mathrm{THz}$ generation systems, and the application of electromagnetic bandgaps to microwave and millimeter wave circuits. He is now very active in the field of metamaterials and their application to the miniaturization and optimization of microwave circuits and antennas. He is the head of the Microwave Engineering, Metamaterials and Antennas Group (GEMMA Group) at UAB, and director of CIMITEC, a research Center on Metamaterials supported by TECNIO (Generalitat de Catalunya). He has organized several international events related to metamaterials, including Workshops at the IEEE International Microwave Symposium (years 2005 and 2007) and European Microwave Conference (2009), and the Fifth International Congress on Advanced Electromagnetic Materials in Microwaves and Optics (Metamaterials 2011), where he has acted as chair of the Local Organizing Committee. He has acted as Guest Editor for three Special Issues on Metamaterials in three International Journals. He has authored and co-authored over 500 technical conference, letter, journal papers and book chapters, he is co-author of the book on Metamaterials entitled Metamaterials with Negative Parameters: Theory, Design and Microwave Applications (John Wiley \& Sons Inc.), author of the book Artificial Transmission Lines for RF and Microwave Applications (John Wiley \& Sons Inc.), and he has generated 17 PhDs. Ferran Martín has filed several patents on metamaterials and has headed several Development Contracts.

Prof. Martín is a member of the IEEE Microwave Theory and Techniques Society (IEEE MTT-S). He is reviewer of the IEEE Transactions on Microwave Theory and Techniques and IEEE Microwave and Wireless Components Letters, among many other journals, and he serves as member of the Editorial Board of IET Microwaves, Antennas and Propagation, International Journal of RF and Microwave Computer-Aided Engineering, and Sensors. He is also a member of the Technical Committees of the European Microwave Conference (EuMC) and International Congress on Advanced Electromagnetic Materials in Microwaves and Optics (Metamaterials). Among his distinctions, Ferran Martín has received the 2006 Duran Farell Prize for Technological Research, he holds the Parc de Recerca UAB - Santander Technology Transfer Chair, and he has been the recipient of two ICREA ACADEMIA Awards (calls 2008 and 2013). 